\title{
INVARIANT DENSITIES OF RANDOM MAPS HAVE LOWER BOUNDS ON THEIR SUPPORTS
}

\author{
PAWEŁ GÓRA, ABRAHAM BOYARSKY, AND MD SHAFIQUL ISLAM
}

Received 8 February 2005; Revised 2 October 2005; Accepted 4 October 2005

A random map is a discrete-time dynamical system in which one of a number of transformations is randomly selected and applied at each iteration of the process. The asymptotic properties of a random map are described by its invariant densities. If Pelikan's average expanding condition is satisfied, then the random map has invariant densities. For individual maps, piecewise expanding is sufficient to establish many important properties of the invariant densities, in particular, the fact that the densities are bounded away from 0 on their supports. It is of interest to see if this property is transferred to random maps satisfying Pelikan's condition. We show that if all the maps constituting the random map are piecewise expanding, then the same result is true. However, if one or more of the maps are not expanding, this may not be true: we present an example where Pelikan's condition is satisfied, but not all the maps are piecewise expanding, and show that the invariant density is not separated from 0 .

Copyright (C 2006 Paweł Góra et al. This is an open access article distributed under the Creative Commons Attribution License, which permits unrestricted use, distribution, and reproduction in any medium, provided the original work is properly cited.

\section{Introduction}

A fundamental problem in ergodic theory is to describe the asymptotic behavior of trajectories defined by a dynamical system. In general, the long term behavior of trajectories of a chaotic dynamical system is unpredictable. Therefore, it is natural to describe the behavior of the system by statistical means. In this approach, one attempts to prove the existence of meaningful invariant measures and determine their ergodic properties. For a single transformation, much is known about the densities of the absolutely continuous invariant measures (acim). For example, it is known that the densities inherit smoothness properties from the map itself (Halfant [7]), that the supports consist of a finite union of intervals, and that the densities are bounded below on their supports (Keller [8] and Kowalski [9]). 
Random dynamical systems provide a useful framework for modeling and analyzing various physical, social, and economic phenomena $[4,12]$. A random dynamical system of special interest is a random map where the process switches from one map to another according to fixed probabilities [11] or, more generally, position-dependent probabilities $[2,6]$. In [4] we model the two-slit experiment of quantum mechanics by a random map. More specifically, given two probability density functions, $f_{1}$ and $f_{2}$, we construct maps $\tau_{1}$ and $\tau_{2}$ which have $f_{1}$ and $f_{2}$ as their respective invariant probability density functions. We then define a random map based on these two maps; that is a discrete-time random process which at each time chooses one map or the other with specified probability. This process is referred to as a random map and possesses an invariant probability density function which is a "combination" of $f_{1}$ and $f_{2}$. Computer experiments on this random map confirm the similarity to the interference results of the two-slit experiment. Random maps are also a convenient framework for modeling processes with randomly changing environment, for example, stock market. We used it in [2] to replace the binomial model applied to determine option prices.

The existence and properties of invariant measures for random maps reflect their long time behavior and play an important role in understanding their chaotic nature. It is, therefore, important to establish properties of their absolutely continuous invariant measures. In this paper we generalize to random maps results of Keller [8] and Kowalski [9], which state that the density of an acim of a nonsingular map is strictly positive on its support. Our main results are proven under the assumption that the individual maps used to construct the random map are piecewise expanding. We also give an example satisfying Pelikan's condition (2.5), showing that the assumption of expanding cannot be removed.

In Section 2 we present the notation and summarize the results we will need in the sequel. In Section 3 we prove the main result.

\section{Preliminaries}

Let $(X, \mathscr{B}, \lambda)$ be a measure space, where $\lambda$ is an underlying measure and $\tau_{k}: X \rightarrow X, k=$ $1,2, \ldots, K$ are nonsingular transformations. A random map $T$ with constant probabilities is defined as

$$
T=\left\{\tau_{1}, \tau_{2}, \ldots, \tau_{K} ; p_{1}, p_{2}, \ldots, p_{K}\right\}
$$

where $\left\{p_{1}, p_{2}, \ldots, p_{K}\right\}$ is a set of constant probabilities. For any $x \in X, T(x)=\tau_{k}(x)$ with probability $p_{k}$ and, for any nonnegative integer $N, T^{N}(x)=\tau_{k_{N}} \circ \tau_{k_{N-1}} \circ \cdots \circ \tau_{k_{1}}(x)$ with probability $\Pi_{j=1}^{N} p_{k_{j}}$. A measure $\mu$ is $T$-invariant if and only if it satisfies the following condition [11]:

$$
\mu(E)=\sum_{k=1}^{K} p_{k} \mu\left(\tau_{k}^{-1}(E)\right),
$$

for any $E \in \mathscr{B}$.

We now recall some definitions and results which will be needed to prove the main result in Section 3. 
Definition 2.1. Let $\tau:(X, \mathscr{B}, \lambda) \rightarrow(X, \mathscr{B}, \lambda)$ be a nonsingular transformation and let $\mu$ be an acim with respect to Lebesgue measure $\lambda$ possessing density function $f$. We define the support of $\mu$ as follows:

$$
\operatorname{supp}(\mu)=\operatorname{supp}(f)=\{x \in X: f(x)>0\}
$$

Definition 2.2. A function $f: \mathbb{R} \rightarrow \mathbb{R}$ is said to be a lower semicontinuous function if and only if $f(y) \leq \liminf _{x \rightarrow y} f(x)$ for any $y \in \mathbb{R}$.

Theorem 2.3 [5]. If $f$ is lower semicontinuous on $I=[a, b] \subset \mathbb{R}$, then it is bounded below and assumes its minimum value. For any $c \in \mathbb{R}$, the set $\{x: f(x)>c\}$ is open.

LEMмA 2.4 [5]. If $f$ is of bounded variation on $I$, then it can be redefined on a countable set to become a lower semicontinuous function.

Let $\mathscr{T}_{0}(I)$ denote the class of transformations $\tau: I \rightarrow I$ that satisfies the following conditions:

(i) $\tau$ is piecewise monotonic, that is, there exists a partition $\mathscr{P}=\left\{I_{i}=\left[a_{i-1}, a_{i}\right], i=\right.$ $1,2, \ldots, q\}$ of $I$ such that $\tau_{i}=\tau \mid I_{i}$ is $C^{1}$, and

$$
\left|\tau_{i}^{\prime}(x)\right| \geq \alpha>0
$$

for any $i$ and for all $x \in\left(a_{i-1}, a_{i}\right)$;

(ii) $g(x)=1 /\left|\tau_{i}^{\prime}(x)\right|$ is a function of bounded variation, where $\tau_{i}^{\prime}(x)$ is the appropriate one-sided derivative at the end points of $\mathscr{P}$.

We say that $\tau \in \mathscr{T}_{1}(I)$ if $\tau \in \mathscr{T}_{0}(I)$ and $\alpha>1$ in condition (2.4), that is, $\tau$ is piecewise expanding.

Theorem $2.5[8,9]$. Let $\tau \in \mathscr{T}_{1}(I)$ and $f$ be a $\tau$-invariant density which can be assumed to be lower semicontinuous. Then there exists a constant $\beta>0$ such that $\left.f\right|_{\operatorname{supp}(f)} \geq \beta$.

Theorem $2.6[1,11]$. Let $T=\left\{\tau_{1}, \tau_{2}, \ldots, \tau_{K} ; p_{1}, p_{2}, \ldots, p_{K}\right\}$ be a random map, where $\tau_{k} \in$ $\mathscr{T}_{0}(I)$, with the common partition $\mathscr{P}=\left\{J_{1}, J_{2}, \ldots, J_{q}\right\}, k=1,2, \ldots, K$. If, for all $x \in[0,1]$, the following Pelikan's condition

$$
\sum_{k=1}^{K} \frac{p_{k}}{\left|\tau_{k}^{\prime}(x)\right|} \leq \gamma<1
$$

is satisfied, then for all $f \in L^{1}=L^{1}([0,1], \lambda)$ :

(i) the limit

$$
\lim _{n \rightarrow \infty} \frac{1}{n} \sum_{i=1}^{n-1} P_{T}^{i}(f)=f^{*} \quad \text { exists in } L^{1}
$$

(ii) $P_{T}\left(f^{*}\right)=f^{*}$;

(iii) $V_{[0,1]}\left(f^{*}\right) \leq C \cdot\|f\|_{1}$, for some constant $C>0$, which is independent of $f \in L^{1}$. 
4 Invariant densities of random maps

\section{Support of invariant density of random maps}

In this section we prove that the invariant density of an acim of the random map $T=$ $\left\{\tau_{1}, \tau_{2}, \ldots, \tau_{K} ; p_{1}, p_{2}, \ldots, p_{K}\right\}, \tau_{1}, \tau_{2}, \ldots, \tau_{K} \in \mathscr{T}_{1}$ is strictly positive on its support. For notational convenience, we consider $K=2$, that is, we consider only two transformations $\tau_{1}, \tau_{2}$. The proofs for larger number of maps are analogous. We consider random maps with constant probabilities.

Let 2 denote the set of endpoints of intervals of partition $\mathscr{P}$ except the points 0 and 1.

The main result of this paper applies to random maps, where each component map is in $\mathscr{T}_{1}(I)$, but the first two lemmas are proved under the more general assumptions of Theorem 2.6.

Lemma 3.1. Let the random map $T=\left\{\tau_{1}, \tau_{2} ; p_{1}, p_{2}\right\}$ satisfy the assumptions of Theorem 2.6. In particular,

$$
\frac{p_{1}}{\left|\tau_{1}^{\prime}(x)\right|}+\frac{p_{2}}{\left|\tau_{2}^{\prime}(x)\right|} \leq \gamma<1
$$

for all $x \in I \backslash$ 2. Then, for any interval $J$ disjoint with 2 , at least one of the images $\tau_{1}(J)$, $\tau_{2}(J)$ is longer than $J$.

Proof. First, let us note that if $v$ is the normalized Lebesgue measure on $J$, then

$$
\begin{gathered}
1=\left(\int_{J} 1 d \nu\right)^{2}=\left(\int_{J} \sqrt{\left|\tau^{\prime}(x)\right|} \cdot \frac{1}{\sqrt{\left|\tau^{\prime}(x)\right|}} d \nu(x)\right)^{2} \\
\leq \int_{J}\left|\tau^{\prime}(x)\right| d \nu(x) \cdot \int_{J} \frac{1}{\left|\tau^{\prime}(x)\right|} d \nu(x), \\
\frac{1}{\int_{J}\left|\tau^{\prime}(x)\right| d \nu(x)} \leq \int_{J} \frac{1}{\left|\tau^{\prime}(x)\right|} d \nu(x),
\end{gathered}
$$

or

$$
\frac{1}{(1 / \lambda(J)) \int_{J}\left|\tau^{\prime}(x)\right| d x} \leq \frac{1}{\lambda(J)} \int_{J} \frac{1}{\left|\tau^{\prime}(x)\right|} d x
$$

Integrating (3.1) over $J$, we obtain

$$
p_{1} \int_{J} \frac{1}{\left|\tau_{1}^{\prime}(x)\right|} d x+p_{2} \int_{J} \frac{1}{\left|\tau_{2}^{\prime}(x)\right|} d x \leq \gamma \cdot \lambda(J),
$$

and, using (3.4), we obtain

$$
\frac{p_{1} \lambda(J)}{\lambda\left(\tau_{1}(J)\right)}+\frac{p_{2} \lambda(J)}{\lambda\left(\tau_{2}(J)\right)} \leq \gamma
$$

Thus, at least one of the numbers $\lambda\left(\tau_{1}(J)\right), \lambda\left(\tau_{2}(J)\right)$ is larger than $\lambda(J)$. 
Corollary 3.2. Under assumptions of Lemma 3.1, if J is an interval disjoint with endpoints of the partition $\mathscr{P}^{(n)}=\mathscr{P} \vee \vee \tau_{j_{n-1}}^{-1} \tau_{j_{n-2}}^{-1} \cdots \tau_{j_{1}}^{-1} \mathscr{P}$ for $n>1$, then

$$
\sum_{\left(j_{n}, j_{n-1}, \ldots, j_{1}\right)} p_{j_{n}} p_{j_{n-1}} \cdots p_{j_{1}} \frac{\lambda(J)}{\lambda\left(\tau_{j_{n}} \tau_{j_{n-1}} \cdots \tau_{j_{1}}(J)\right)} \leq \gamma^{n}
$$

where $j_{n}, j_{n-1}, \ldots, j_{1} \in\{1,2\}$. In particular, we have

$$
\max _{J \in \mathscr{P}(n)} \operatorname{diam}(J) \leq \gamma^{n}, \quad n \geq 1 .
$$

Proof. It follows from the fact that Pelikan's condition (2.5) implies

$$
\sup _{x} \sum_{\left(j_{n}, j_{n-1}, \ldots, j_{1}\right)} \frac{p_{j_{n}} p_{j_{n-1}} \cdots p_{j_{1}}}{\left|\left(\tau_{j_{n}} \circ \tau_{j_{n-1}} \circ \cdots \circ \tau_{j_{1}}\right)^{\prime}(x)\right|} \leq \gamma^{n}
$$

$n \geq 1, j_{n}, j_{n-1}, \ldots, j_{1} \in\{1,2\}$.

Remark 3.3. Instead of Pelikan's condition (2.5) we can use throughout the paper a weaker condition

$$
p_{1} \ln \left|\tau_{1}^{\prime}(x)\right|+p_{2} \ln \left|\tau_{2}^{\prime}(x)\right| \geq \beta>0, \quad x \in[0,1],
$$

which one could call Morita's condition [10]. Condition (3.10) implies the existence of absolutely continuous invariant measure but its density may not be of bounded variation, see [3, Example 1.2]. If we assume additionally that this density is of bounded variation or at least is a lower semicontinuous function, then we can reprove the results of this paper under assumption of condition (3.10).

Condition (3.10) implies

$$
\sum_{\left(j_{n}, j_{n-1}, \ldots, j_{1}\right)} p_{j_{n}} p_{j_{n-1}} \cdots p_{j_{1}} \ln \left|\left(\tau_{j_{n}} \circ \tau_{j_{n-1}} \circ \cdots \circ \tau_{j_{1}}\right)^{\prime}(x)\right| \geq n \beta>0, \quad x \in[0,1],
$$

$n \geq 1, j_{n}, j_{n-1}, \ldots, j_{1} \in\{1,2\}$. Using condition (3.10) we can prove an analogue of Lemma 3.1 .

For any interval $J$ contained in an element of partition $\mathscr{P}$, we have

$$
p_{1} \ln \frac{\lambda\left(\tau_{1}(J)\right)}{\lambda(J)}+p_{2} \ln \frac{\lambda\left(\tau_{2}(J)\right)}{\lambda(J)} \geq \beta>0
$$

which implies that at least one of the images is longer than interval $J$.

Analogously, for any interval $J$ contained in an element of partition $\mathscr{P}^{(n)}$, we have

$$
\sum_{\left(j_{n}, j_{n-1}, \ldots, j_{1}\right)} p_{j_{n}} p_{j_{n-1}} \cdots p_{j_{1}} \ln \frac{\lambda\left(\tau_{j_{n}} \circ \tau_{j_{n-1}} \circ \cdots \circ \tau_{j_{1}}(J)\right)}{\lambda(J)} \geq n \beta>0,
$$

$n \geq 1, j_{n}, j_{n-1}, \ldots, j_{1} \in\{1,2\}$. This, in particular, implies that

$$
\max _{J \in \mathscr{P}^{(n)}} \operatorname{diam}(J) \leq e^{-n \beta}, \quad n \geq 1 .
$$


Lemma 3.4. Let $T=\left\{\tau_{1}, \tau_{2} ; p_{1}, p_{2}\right\}$ be a random map on $[0,1]$ satisfying the conditions of Theorem 2.6. Then, the support of the invariant density of $T$ contains an interval which is not disjoint with 2.

Proof. Let $\operatorname{supp}(f)=\{x \in[0,1]: f(x)>0\}$. The density function $f$ of the acim $\mu$ is a function of bounded variation by Theorem 2.6 and thus, by Lemma 2.4, $f$ can be redefined on a countable set to become a lower semicontinuous function $\bar{f}$ and $\bar{f}=f$ almost everywhere. Thus, $\operatorname{supp}(f)=\operatorname{supp}(\bar{f})=\{x: \bar{f}>0\}$ is an open set by Theorem 2.3. Thus, $\operatorname{supp}(f)=\cup_{i=1}^{\infty} I_{i}$, where $I_{i}$ 's are open disjoint intervals. Without loss of generality, let us assume that $\lambda\left(I_{i}\right) \geq \lambda\left(I_{i+1}\right)$ for $i=1,2, \ldots$. We will prove that $2 \cap I_{1} \neq \varnothing$. Suppose $2 \cap I_{1}=\varnothing$. Then $I_{1}$ is contained in one of the subintervals, $J_{*}$, of the partition $\mathscr{P}$ and $\tau_{1}\left(I_{1}\right)$ and $\tau_{2}\left(I_{1}\right)$ are both open intervals. Since $f$ is an invariant density of the random map $T$, we have

$$
f(x)=p_{1} \cdot \sum_{i=1}^{q} \frac{f\left(\tau_{1, i}^{-1}(x)\right)}{\left|\tau_{1}^{\prime}\left(\tau_{1, i}^{-1}(x)\right)\right|} \chi_{\tau_{1}\left(I_{i}\right)}(x)+p_{2} \cdot \sum_{i=1}^{q} \frac{f\left(\tau_{2, i}^{-1}(x)\right)}{\left|\tau_{2}^{\prime}\left(\tau_{2, i}^{-1}(x)\right)\right|} \chi_{\tau_{2}\left(I_{i}\right)}(x) .
$$

Let $x \in \tau_{1}\left(I_{1}\right)$. It is clear that at least one element of $\left\{\tau_{1, *}^{-1}(x)\right\}$ is in $I_{1}$ and since $I_{1} \subset$ $\operatorname{supp}(f)$ we have $f(x)>0$. Thus, $\tau_{1}\left(I_{1}\right)$ is a subset of $\operatorname{supp}(f)$. Similarly, $\tau_{2}\left(I_{1}\right)$ is a subset of $\operatorname{supp}(f)$. By Lemma 3.1, at least one of the intervals $\tau_{1}\left(I_{1}\right), \tau_{2}\left(I_{1}\right)$ has larger length than the length of $I_{1}$. This is a contradiction because $\operatorname{supp}(f)$ does not contain an interval of length greater than $\lambda\left(I_{1}\right)$. This proves that $2 \cap I_{1} \neq \varnothing$.

COROLlary 3.5. The number of different ergodic acim for the random map $T$ satisfying the assumptions of Theorem 2.6 is at most equal to the cardinality of the partition $\mathscr{P}$ minus one.

For Theorem 3.6 we assume that $\tau_{1}, \tau_{2} \in \mathscr{T}_{1}$. After the theorem we give an example showing that it fails if we assume only $\tau_{1}, \tau_{2} \in \mathscr{T}_{0}$.

Theorem 3.6. Let $T=\left\{\tau_{1}, \tau_{2} ; p_{1}, p_{2}\right\}$ be a random map on $[0,1]$, where $\tau_{1}, \tau_{2} \in \mathscr{T}_{1}$ and have a common partition $\mathscr{P}=\left\{J_{1}, J_{2}, \ldots, J_{q}\right\}$. Then the support of the invariant density $f$ of $T, \operatorname{supp}(f)$ is a finite union of open intervals almost everywhere.

Proof. Again we can assume that $\operatorname{supp}(f)=\cup_{i=1}^{\infty} I_{i}$, where $I_{i}$ 's are open disjoint intervals. Let $\mathscr{D}=\left\{j \geq 1: I_{j}\right.$ contains a discontinuity of $\tau_{1}, \tau_{2}$, or both $\}$. By Lemma 3.4, $\mathscr{D}$ is not empty. Also, $\mathscr{D}$ is a finite set. If $j \in \mathscr{D}$, then $\tau_{i}\left(I_{j}\right), i=1,2$ is a finite union of intervals. Let $J$ be the shortest interval of the family

$$
\left\{I_{j}\right\}_{j \in \mathscr{D}} \cup\left\{I: I \text { is a connected component of } \tau_{i}\left(I_{j}\right), i=1,2, j \in \mathscr{D}\right\} \text {. }
$$

Let $\mathscr{F}=\left\{i \geq 1: \lambda\left(I_{i}\right) \geq \lambda(J)\right\}$, where $i$ is not necessarily in $\mathscr{D}$ and let

$$
S=\cup_{i \in \mathscr{F}} I_{i} \subset \cup_{i} I_{i}=\operatorname{supp}(f) .
$$

$S$ is a finite union of open disjoint intervals since it is a family of disjoint intervals with length $\geq \lambda(J)>0$. For any $j \in \mathscr{D}, I_{j} \subseteq S$. 
We will prove that $\tau_{i}(S) \subseteq S, i=1,2$. Let $I_{k} \subset S$. If $k \notin \mathscr{D}$, then $\tau_{i}\left(I_{k}\right)$ is contained in an interval $I_{k_{i}}, i=1,2$ and

$$
\lambda\left(I_{k_{i}}\right) \geq \lambda\left(\tau_{i}\left(I_{k}\right)\right)=\int_{I_{k}}\left|\tau_{i}^{\prime}(x)\right| d \lambda>\inf _{x \in[0,1]}\left|\tau_{i}^{\prime}(x)\right| \cdot \lambda\left(I_{k}\right), \quad i=1,2 .
$$

Since $\inf _{x \in[0,1]}\left|\tau_{i}^{\prime}(x)\right|>1$ for $i=1,2$, we have

$$
\lambda\left(I_{k_{i}}\right) \geq \lambda\left(I_{k}\right)>\lambda(J) .
$$

Thus, by the definition of $S$, we get $I_{k_{i}} \subseteq S, i=1,2$ and hence $\tau_{i}\left(I_{k}\right) \subset I_{k_{i}} \subseteq S, i=1,2$. If $k \in \mathscr{D}$, then by the definition of $S, \tau_{i}\left(I_{k}\right) \subset S, i=1,2$. Thus, $\tau_{i}(S) \subseteq S, i=1,2$.

Now we will prove that $\operatorname{supp}(f) \subseteq S$. Suppose not. Let $I_{S}$ be the largest interval of $\operatorname{supp}(f) \backslash S$. Thus, $s \notin \mathscr{D}$ and

$$
\lambda\left(\tau_{i}\left(I_{s}\right)\right)=\int_{I_{s}}\left|\tau_{i}^{\prime}(x)\right| d \lambda>\inf _{x \in[0,1]}\left|\tau_{i}^{\prime}(x)\right| \cdot \lambda\left(I_{s}\right)>\lambda\left(I_{s}\right), \quad i=1,2
$$

Then $\tau_{i}\left(I_{s}\right) \subset S, i=1,2$. Thus, $I_{s} \subset \tau_{i}^{-1}(S), i=1,2$. But $I_{s} \not \subset S$, so $I_{s} \subset \tau_{i}^{-1}(S) \backslash S, i=$ 1,2 . Let $\mu=f \cdot \lambda$ be the $T$-invariant absolutely continuous measure. We will show that $\mu\left(\tau_{i}^{-1}(S) \backslash S\right)=0, i=1,2$. Since $\tau_{i}(S) \subseteq S, i=1,2$, we have

$$
S \subseteq \tau_{i}^{-1}\left(\tau_{i}(S)\right) \subseteq \tau_{i}^{-1}(S), \quad i=1,2
$$

Thus,

$$
\begin{aligned}
0 & =\mu(S)-\mu(S)=\left[p_{1} \mu\left(\tau_{1}^{-1} S\right)+p_{2} \mu\left(\tau_{2}^{-1} S\right)\right]-\left[p_{1} \mu(S)+p_{2} \mu(S)\right] \\
& =p_{1}\left[\mu\left(\tau_{1}^{-1} S\right)-\mu(S)\right]+p_{2}\left[\mu\left(\tau_{2}^{-1} S\right)-\mu(S)\right] \\
& =p_{1} \mu\left(\tau_{1}^{-1} S \backslash S\right)+p_{2} \mu\left(\tau_{2}^{-1} S \backslash S\right) .
\end{aligned}
$$

Thus, both $\mu\left(\tau_{1}^{-1} S \backslash S\right)=0$ and $\mu\left(\tau_{2}^{-1} S \backslash S\right)=0$ if $p_{1}, p_{2}>0$. Thus, $\mu\left(I_{s}\right)=0$, which contradicts the fact that $I_{s} \subset \operatorname{supp}(f)$.

Example 3.7. We present a random map satisfying the assumptions of Theorem 2.6, where the support of absolutely continuous $T$-invariant measure is an infinite countable union of disjoint intervals. In this case the invariant density cannot be bounded away from 0 on its support. Let us define the maps

$$
\begin{aligned}
& \tau_{1}(x)=\frac{(8 x-E(8 x))}{4}, \\
& \tau_{2}(x)= \begin{cases}\frac{3}{4}+\frac{x}{2} & \text { for } 0 \leq x \leq \frac{1}{2}, \\
\frac{x}{2} & \text { for } \frac{1}{2}<x \leq 1,\end{cases}
\end{aligned}
$$

where $E(t)$ denotes the integral part of $t$, as shown in Figure 3.1. 


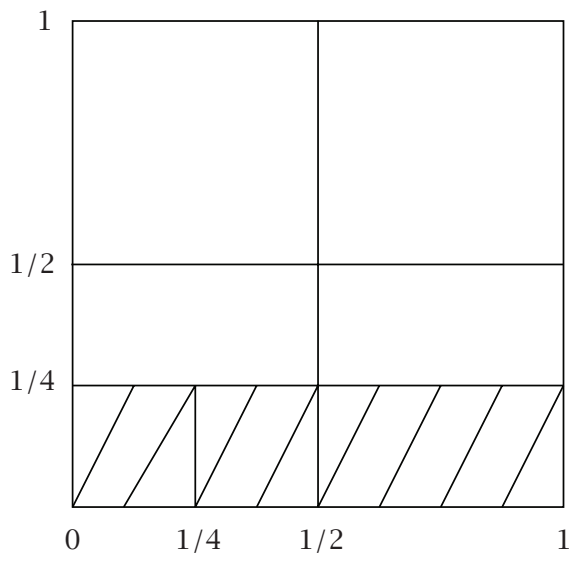

(a)

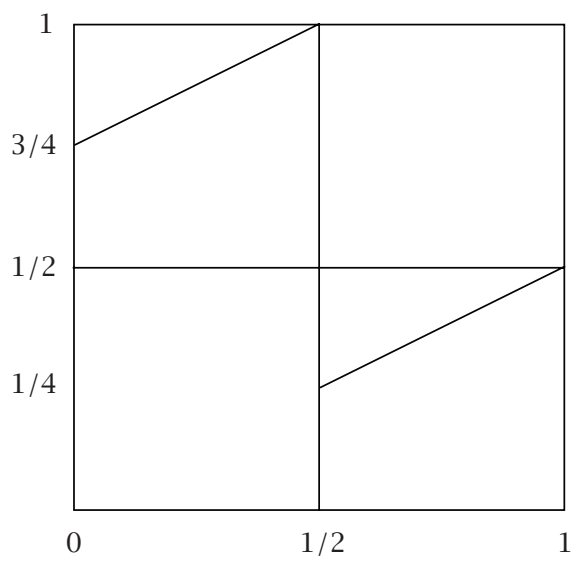

(b)

Figure 3.1. The graphs of $\tau_{1}$ and $\tau_{2}$.

Let us consider the random map $T=\left\{\tau_{1}, \tau_{2} ; 3 / 4,1 / 4\right\}$. It satisfies all the assumptions of Pelikan's Theorem 2.6. In particular, $T$ admits an absolutely continuous invariant measure $\mu$ with density $f$ of bounded variation. Let $S$ denote the support of $f$. Lebesgue measure restricted to the interval $L_{0}=[0,1 / 4]$ is invariant for $\tau_{1}$. Since $\tau_{1}([0,1])=L_{0}$ and $\tau_{1}$ is exact on $L_{0}$, we have $L_{0} \subset S$. Since the interval $K_{0}=(1 / 4,1 / 2)$ is not in the image of $\tau_{1}$ or $\tau_{2}$, it is disjoint from $S$. Every image of $L_{0}$ is in $S$. Since $\tau_{1}([0,1])=L_{0}$, we consider only images by $\tau_{2}$. We have

$$
\begin{aligned}
\tau_{2}\left(L_{0}\right) & =[1-1 / 4,1-1 / 8]=L_{1}^{(1)}, \\
\tau_{2}\left(L_{1}^{(1)}\right) & =[1 / 2-1 / 8,1 / 2-1 / 16]=L_{1}^{(2)}, \\
\tau_{2}\left(L_{1}^{(2)}\right) & =[1-1 / 16,1-1 / 32]=L_{2}^{(1)}, \\
\tau_{2}\left(L_{2}^{(1)}\right) & =[1 / 2-1 / 32,1 / 2-1 / 64]=L_{2}^{(2)},
\end{aligned}
$$

and in general, for $L_{n}^{(1)}=\left[1-1 / 4^{n}, 1-1 /\left(2 \cdot 4^{n}\right)\right]$ and $L_{n}^{(2)}=\left[1 / 2-1 /\left(2 \cdot 4^{n}\right), 1 / 2-1 /(4 \cdot\right.$ $\left.4^{n}\right)$,

$$
\tau_{2}\left(L_{n}^{(1)}\right)=L_{n}^{(2)}, \quad \tau_{2}\left(L_{n}^{(2)}\right)=L_{n+1}^{(1)}
$$

Let $K_{n}^{(1)}$ be the open interval between $L_{n}^{(1)}$ and $L_{n+1}^{(1)}$, and let $K_{n}^{(2)}$ be the open interval between $L_{n}^{(2)}$ and $L_{n+1}^{(2)}, n \geq 1$. Then, obviously, we have for all $n \geq 1$,

$$
\tau_{2}\left(K_{n}^{(1)}\right)=K_{n}^{(2)}, \quad \tau_{2}\left(K_{n}^{(2)}\right)=K_{n+1}^{(1)} .
$$


Since $\tau_{2}$ is an injective map, we also have

$$
\begin{gathered}
\tau_{2}^{-1}\left(K_{n}^{(2)}\right)=K_{n}^{(1)}, \\
\tau_{2}^{-1}\left(K_{n+1}^{(1)}\right)=K_{n}^{(2)}, \quad n \geq 1 .
\end{gathered}
$$

We have

$$
\tau_{2}^{-2}\left(K_{1}^{(1)}\right)=K_{0}
$$

and $\tau_{1}^{-1}\left(K_{n}^{(i)}\right)=\varnothing$ for $i=1,2, n \geq 1$. Thus,

$$
\left(K_{n}^{(i)}\right) \cap S=\varnothing, \quad i=1,2 ; n \geq 1 .
$$

We have proved that

$$
S=L_{0} \cup \bigcup_{i=1,2} \bigcup_{n \geq 1} L_{n}^{(i)}
$$

that is, the support of the absolutely continuous $T$-invariant measure is an infinite countable union of disjoint intervals. Since the invariant density $f$ is of bounded variation, we have

$$
\sup _{x \in L_{n}^{(i)}} f(x) \longrightarrow 0, \quad n \longrightarrow+\infty
$$

for $i=1,2$, so the density $f$ is not bounded away from 0 .

In Lemma 3.8 and Theorem 3.9, we return to the assumption $\tau_{1}, \tau_{2} \in \mathscr{T}_{0}$, but we assume additionally that the support of the invariant density is a finite union of disjoint intervals. That was proved in Theorem 3.6 using the assumption $\tau_{1}, \tau_{2} \in \mathscr{T}_{1}$.

Lemma 3.8. Let $T=\left\{\tau_{1}, \tau_{2} ; p_{1}, p_{2}\right\}$ be a random map on $[0,1]$, where $\tau_{1}, \tau_{2} \in \mathscr{T}_{0}$ and have a common partition $\mathscr{P}=\left\{J_{1}, J_{2}, \ldots, J_{q}\right\}$. Let $f$ be the invariant density of an acim $\mu$ of the random map $T$ and $S=\operatorname{supp}(f)=\{x: f(x)>0\}$. We assume that $S$ is a finite union of disjoint intervals. Then

(i) $\tau_{i}\left(S \backslash\left\{a_{0}, a_{1}, \ldots, a_{q}\right\}\right) \subseteq S, i=1,2$;

(ii) $\lambda\left(S \backslash \tau_{i}\left(S \backslash\left\{a_{0}, a_{1}, \ldots, a_{q}\right\}\right)\right)=0, i=1,2$;

where $\left\{a_{0}, a_{1}, \ldots, a_{q}\right\}$ are the endpoints of the intervals in the partition $\mathscr{P}$.

Proof. We assume that $f$ is lower semicontinuous. If it is not, we modify it on at most a countable set. By assumption, $S=\cup_{i=1}^{r} I_{i}$. Let $x \in S \backslash\left\{a_{0}, a_{1}, \ldots, a_{q}\right\}$. Then $x \in \operatorname{Int} I_{k}$, for some $k \in\{1,2, \ldots, r\}$ and there exists $\epsilon>0$ such that $B(x, \epsilon) \subset I_{k}$ and $f(y)>(1 / 2) f(x)>$ 0 for all $y \in B(x, \epsilon)$ since $f$ is lower semicontinuous. We may assume that $\tau_{i} \mid I_{k}, i=1,2$ is 
increasing and that $f\left(\tau_{i}(x)\right)=\lim _{y \rightarrow \tau_{i}(x)^{+}} f(y), i=1,2$. Now, for any $\delta>0, \tau_{i}([x, x+\delta))=$ $\left[\tau_{i}(x), \tau_{i}(x)+\delta^{\prime}\right), i=1,2$ and $\delta^{\prime} \rightarrow 0$ as $\delta \rightarrow 0$. Then, for $i=1,2$, we have

$$
\begin{aligned}
\int_{\left[\tau_{i}(x), \tau_{i}(x)+\delta^{\prime}\right)} f d \lambda & =\mu\left(\tau_{i}([x, x+\delta))\right) \geq \mu([x, x+\delta)) \\
& =\int_{[x, x+\delta)} f d \lambda \geq \frac{1}{2} f(x) \lambda([x, x+\delta)) \\
& \geq \frac{1}{2} f(x) \frac{1}{\max \left|\tau_{i}^{\prime}\right|} \lambda\left(\left[\tau_{i}(x), \tau_{i}(x)+\delta^{\prime}\right)\right) .
\end{aligned}
$$

Since $f$ is lower semicontinuous,

$$
\begin{aligned}
f\left(\tau_{i}(x)\right) & =\lim _{\delta^{\prime} \rightarrow 0} \frac{1}{\lambda\left(\left[\tau_{i}(x), \tau_{i}(x)+\delta^{\prime}\right)\right)} \int_{\left[\tau_{i}(x), \tau_{i}(x)+\delta^{\prime}\right)} f d \lambda \\
& \geq \frac{1}{2} f(x) \frac{1}{\max \left|\tau_{i}^{\prime}\right|}>0, \quad i=1,2 .
\end{aligned}
$$

Hence, $\tau_{i}(x) \in S, i=1,2$, and part (i) is proved.

Part (ii) is proved using reasoning similar to the end of Theorem 3.6 (3.22), which does not depend on the expanding property of $\tau_{1}, \tau_{2}$.

Theorem 3.9. Let $T=\left\{\tau_{1}, \tau_{2} ; p_{1}, p_{2}\right\}$ be a random map on $[0,1]$, where $\tau_{1}, \tau_{2} \in \mathscr{T}_{1}$ and have a common partition $\mathscr{P}=\left\{J_{1}, J_{2}, \ldots, J_{q}\right\}$. Let $f$ be the invariant density of an acim $\mu$ of the random map $T$ and let $S=\operatorname{supp} f=\{x: f(x)>0\}$. We assume that $S$ is a finite union of disjoint intervals. Then there exists a constant $a>0$ such that $f_{\mid s} \geq a$.

Proof. Since $S=\{x: f(x)>0\}$ is a finite union of open intervals, $S=\cup_{i=1}^{r} I_{i}$, we can assume they are separated by intervals of positive measure. Then $\widehat{S}=S \backslash\left\{a_{0}, a_{1}, \ldots, a_{q}\right\}$ is also a finite union of intervals: $\widehat{S}=\cup_{i=1}^{s} J_{i}$. Let $\mathscr{F}=\left\{I_{i}\right\}_{i=1}^{r}$, and let $\mathscr{C}=\left\{J_{j}\right\}_{j=1}^{s}$. For any $J_{k} \in \mathscr{C},\left.\tau_{j}\right|_{J_{k}}, j=1,2$ is of class $C^{1}$. Therefore, there exist $I_{i_{j}} \in \mathscr{F}, j=1,2$ such that $\tau_{j}\left(J_{k}\right) \subseteq I_{i_{j}}, j=1,2$.

Let $(c, d)$ be any interval in $\mathscr{F}$ or $\mathscr{C}$. We associate with its endpoints two classes of standard intervals:

$$
\eta_{c}=\{(c, c+\epsilon): \epsilon>0\}, \quad \eta_{d}=\{(d-\epsilon, d): \epsilon>0\} .
$$

The points $c$ and $d$ are referred to as the endpoints of the classes $\eta_{c}$ and $\eta_{d}$, respectively. Let

$$
\mathscr{K}=\left\{\eta_{c}, \eta_{d}: c, d \text { are endpoints of intervals of } \mathscr{F}, \mathscr{C}\right\}
$$

We now define a relation $\mapsto$ between elements of $\mathscr{K}$. For $\eta, \eta^{\prime} \in \mathcal{K}: \eta \mapsto \eta^{\prime}$ if and only if $\tau_{j}(U) \in \eta^{\prime}$ for at least one of $j=1,2$ and for sufficiently small $U \in \eta$. The relation has the following two properties:

(1) if $\eta^{\prime}$ is associated with an endpoint of $I_{i} \in \mathscr{F}_{F}$, then there exists at least one $\eta$ such that $\eta \mapsto \eta^{\prime}$. To prove this, let us fix $I_{i}$ and $\eta^{\prime}$ associated with one of its endpoints. We 
claim that for each $J_{k} \in \mathscr{C}$, either $\tau_{j}\left(J_{k}\right) \subseteq I_{i}, j=1,2$ or $\tau_{j}\left(J_{k}\right) \cap I_{i}=\varnothing, j=1,2$. To show this, let us note that since $\tau_{j}\left(J_{k}\right), j=1,2$ is contained in $S=\cup_{i=1}^{r} I_{i}$ and $\left\{I_{i}\right\}$ are separated, $\tau_{j}\left(J_{k}\right), j=1,2$ is contained in one of $I_{i_{j}}, j=1,2$. Now, since

$$
\lambda\left(I_{i_{j}} \backslash \tau_{j}(\widehat{S})\right) \leq \lambda\left(S \backslash \tau_{j}(\widehat{S})\right)=0, \quad j=1,2,
$$

there must exist $J_{k}$ with $\tau_{j}\left(J_{k}\right) \in \eta^{\prime}, j=1,2$ and this implies (1);

(2) if $c^{\prime}$ is an endpoint of $I_{i}$ such that $\lim _{x \rightarrow c^{\prime}} f(x)=0, \eta^{\prime}$ is associated with $c^{\prime}, \eta \mapsto \eta^{\prime}$, and $c$ is an endpoint of $\eta$, then for any $U \in \eta$,

$$
\lim _{\substack{x \rightarrow c \\ x \in U}} f(x)=0 .
$$

To prove this let us suppose that $\lim _{x \in U, x \rightarrow c} f(x)=a>0$, for some $U \in \eta$. By the definition of the relation $\mapsto$, for at least one of $j=1,2$, say $j=1$, if $\eta=\{(c, c+\varepsilon)\}$, then we have $\tau_{1}(c, c+\varepsilon)=\left(c^{\prime}, c+\varepsilon^{\prime}\right)$, for $\varepsilon$ small enough. Then,

$$
\begin{aligned}
\lim _{\substack{x \rightarrow c^{\prime} \\
x \in U^{\prime}}} f(x) & =\lim _{\varepsilon \rightarrow 0} \frac{1}{\lambda\left(\left(c^{\prime}, c^{\prime}+\varepsilon^{\prime}\right)\right)} \int_{\left(c^{\prime}, c^{\prime}+\varepsilon^{\prime}\right)} f(t) d t=\lim _{\varepsilon \rightarrow 0} \frac{\mu\left(\left(c^{\prime}, c^{\prime}+\varepsilon^{\prime}\right)\right)}{\lambda\left(\left(c^{\prime}, c^{\prime}+\varepsilon^{\prime}\right)\right)} \\
& \geq \lim _{\varepsilon \rightarrow 0} p_{1} \cdot \frac{\mu((c, c+\varepsilon))}{\max \left|\tau_{1}^{\prime}\right| \cdot \lambda((c, c+\varepsilon))}=\frac{p_{1}}{\max \left|\tau_{1}^{\prime}\right|} a>0,
\end{aligned}
$$

which is a contradiction.

We make the following observations:

(3) in the setting of (2) above, $c \notin S$. Therefore, $c$ is an endpoint of an interval $I_{i} \in \mathscr{F}_{\text {. }}$. Now we define

$$
\mathscr{K}_{0}=\left\{\eta: \eta \text { is associated with an endpoint } c \text { of } I_{i} \in \mathscr{F}, \lim _{\substack{x \rightarrow c \\ x \in I_{i}}} f(x)=0\right\} .
$$

From (2) and (3) we obtain what follows:

(4) if $\eta^{\prime} \in \mathscr{K}_{0}$ and $\eta \mapsto \eta^{\prime}$, then $\eta \in \mathscr{K}_{0}$.

We note that by (1) and (4), for each $\eta^{\prime} \in \mathscr{K}_{0}$ there exists at least one $\eta \in \mathscr{K}_{0}$ such that $\eta \mapsto \eta^{\prime}$.

Now let $\eta \in \mathscr{K}_{0}$. For any $n \geq 1$, any $\eta^{\prime}$ such that $\eta^{\prime} \mapsto \eta$ in $n$-steps also belongs to $\mathscr{K}_{0}$. Choose $U \in \eta$ to be sufficiently small, that is, completely contained in an element of partition $\mathscr{P}$. Then, all the preimages $\tau_{j_{n}}^{-1} \circ \tau_{j_{n-1}}^{-1} \circ \cdots \circ \tau_{j_{2}}^{-1} \circ \tau_{j_{1}}^{-1}(U), j_{i} \in\{1,2\}$ touch an endpoint of some $\eta^{\prime}$ in $\mathscr{K}_{0}$. Let $M$ be the number of elements in $\mathscr{K}_{0}$ and let $\gamma<1$ be the constant from Pelikan's condition (2.5). Then,

$$
\begin{aligned}
\mu(U) & =\sum_{\left(j_{n}, j_{n-1}, \ldots, j_{1}\right)} p_{j_{n}} p_{j_{n-1}} \cdots p_{j_{1}} \mu\left(\tau_{j_{n}}^{-1} \circ \tau_{j_{n-1}}^{-1} \circ \cdots \circ \tau_{j_{1}}^{-1}(U)\right) \\
& \leq \sup f \cdot \sum_{\left(j_{n}, j_{n-1}, \ldots, j_{1}\right)} p_{j_{n}} p_{j_{n-1}} \cdots p_{j_{1}} \lambda\left(\tau_{j_{n}}^{-1} \circ \tau_{j_{n-1}}^{-1} \circ \cdots \circ \tau_{j_{1}}^{-1}(U)\right) .
\end{aligned}
$$


Each $\tau_{j_{n}}^{-1} \circ \tau_{j_{n-1}}^{-1} \circ \cdots \circ \tau_{j_{1}}^{-1}(U)$ consists of intervals contained in elements of partition $\mathscr{P}^{(n)}$ and touches one of $M$ endpoints of classes in $\mathcal{K}_{0}$. Thus, using Corollary 3.2, we have

$$
\begin{gathered}
\lambda\left(\tau_{j_{n}}^{-1} \circ \tau_{j_{n-1}}^{-1} \circ \cdots \circ \tau_{j_{2}}^{-1} \circ \tau_{j_{1}}^{-1}(U)\right) \leq M \cdot \gamma^{n}, \\
\mu(U) \leq \sup f \cdot \sum_{\left(j_{n}, j_{n-1}, \ldots, j_{1}\right)} p_{j_{n}} p_{j_{n-1}} \cdots p_{j_{1}} M \gamma^{n}=\sup f \cdot M \gamma^{n} .
\end{gathered}
$$

Thus, $\mu(U)=0$ which implies that $\lambda(U)=0$ since $U \subseteq S$. This contradicts the fact that $U \in \eta$ is an open, nonempty interval. Hence, $\mathscr{K}_{0}=\varnothing$ and $\lim _{x \in U, x \rightarrow c} f(x)>0$ for each of finitely many endpoints of intervals $I_{i} \in \mathscr{F}$. On the other hand, since $f$ is lower semicontinuous, it assumes its infimum on any closed interval. Hence, there exists $a>0$ such that $f(x) \geq a$ for all $x \in S$.

\section{Acknowledgments}

The authors are grateful to Martijn de Vries who suggested the problem of strict positivity of invariant density of random map. P. Góra is grateful for valuable comments to the participants of International Conference in Dynamical Systems, in memory of Wiesław Szlenk, June 6-11, 2005, Banach Center, Warsaw, Poland. The research was supported by NSERC Grant. M. S. Islam was supported by the Department of Mathematics and Statistics, Concordia University.

\section{References}

[1] W. Bahsoun and P. Góra, Position dependent random maps in one and higher dimensions, Studia Mathematica 166 (2005), no. 3, 271-286.

[2] W. Bahsoun, P. Góra, and A. Boyarsky, Markov switching for position dependent random maps with application to forecasting, SIAM Journal on Applied Dynamical Systems 4 (2005), no. 2, 391-406.

[3] M. Blank, Perron-Frobenius spectrum for random maps and its approximation, Moscow Mathematical Journal 1 (2001), no. 3, 315-344, 470.

[4] A. Boyarsky and P. Góra, A dynamical system model for interference effects and the two-slit experiment of quantum physics, Physics Letters A 168 (1992), no. 2, 103-112.

[5] __ Laws of Chaos, Probability and Its Applications, Birkhäuser Boston, Massachusetts, 1997.

[6] P. Góra and A. Boyarsky, Absolutely continuous invariant measures for random maps with position dependent probabilities, Journal of Mathematical Analysis and Applications 278 (2003), no. 1, $225-242$.

[7] M. Halfant, Analytic properties of Rényi's invariant density, Israel Journal of Mathematics 27 (1977), no. 1, 1-20.

[8] G. Keller, Piecewise monotonic transformations and exactness, Seminar on Probability, Rennes 1978 (French); Exp. No. 6, University of Rennes, Rennes, 1978, p. 32.

[9] Z. S. Kowalski, Invariant measure for piecewise monotonic transformation has a positive lower bound on its support, L'Académie Polonaise des Sciences. Bulletin. Série des Sciences Mathématiques 27 (1979), no. 1, 53-57.

[10] T. Morita, Asymptotic behavior of one-dimensional random dynamical systems, Journal of the Mathematical Society of Japan 37 (1985), no. 4, 651-663. 
[11] S. Pelikan, Invariant densities for random maps of the interval, Transactions of the American Mathematical Society 281 (1984), no. 2, 813-825.

[12] K. R. Schenk-Hoppe, Random dynamical systems in economics, Working Paper Series ISSN 14240459, Institute of Empirical Research in Economics, University of Zurich, Zurich, December 2000.

Paweł Góra: Department of Mathematics and Statistics, Concordia University,

7141 Sherbrooke Street West, Montreal, Quebec, Canada H4B 1R6

E-mail address: pgora@vax2.concordia.ca

Abraham Boyarsky: Department of Mathematics and Statistics, Concordia University,

7141 Sherbrooke Street West, Montreal, Quebec, Canada H4B 1R6

E-mail address: boyar@alcor.concordia.ca

Md Shafiqul Islam: Department of Mathematics and Computer Science, University of Lethbridge, 4401 University Drive, Lethbridge, Alberta, Canada T1K 3M4

E-mail address: islam@cs.uleth.ca 


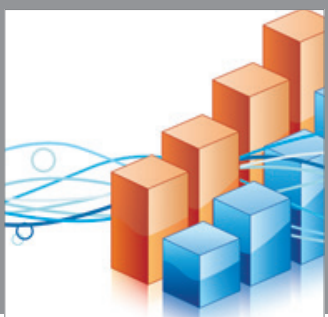

Advances in

Operations Research

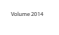

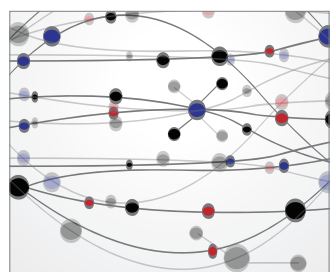

\section{The Scientific} World Journal
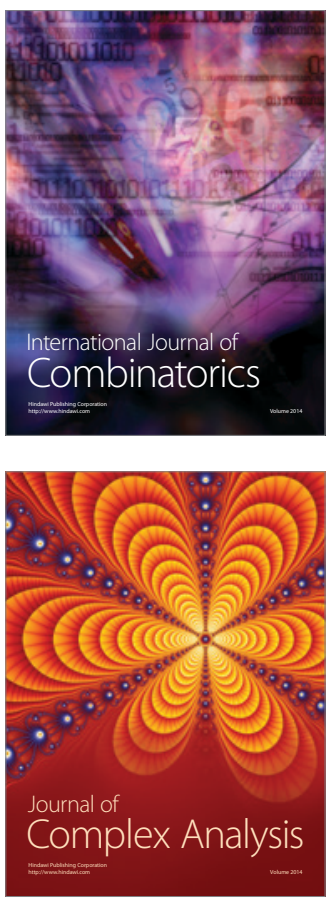

International Journal of

Mathematics and

Mathematical

Sciences
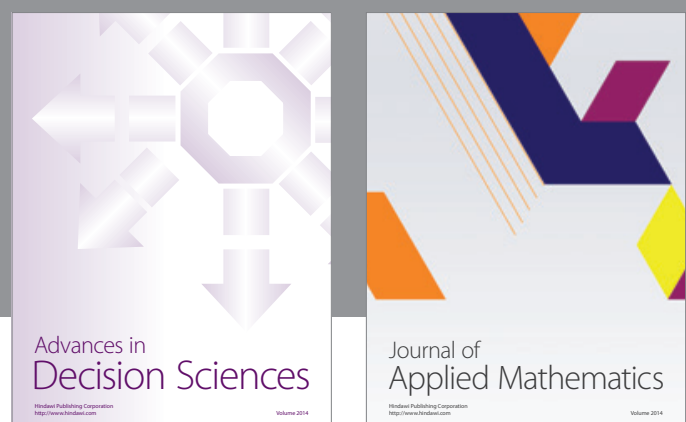

Journal of

Applied Mathematics
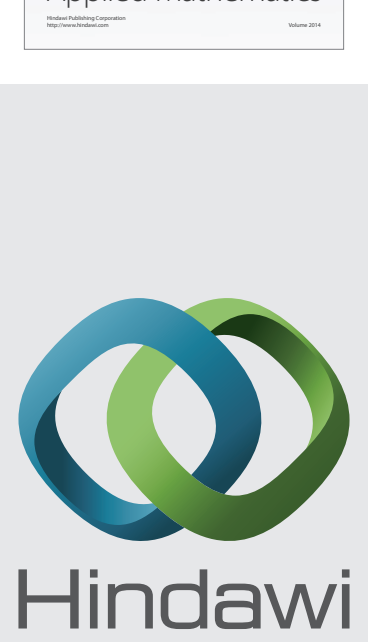

Submit your manuscripts at http://www.hindawi.com
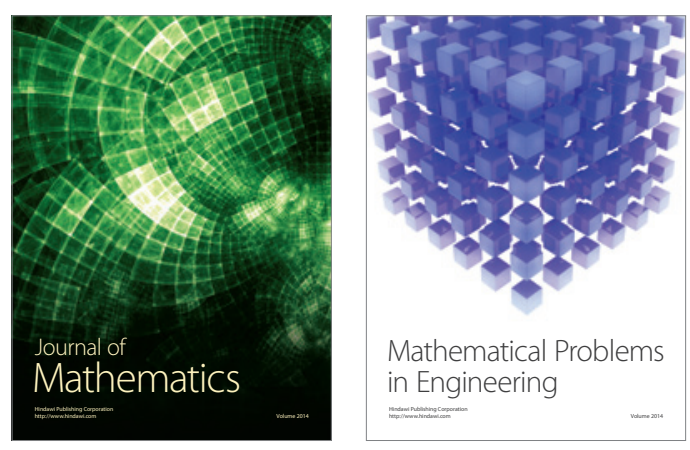

Mathematical Problems in Engineering
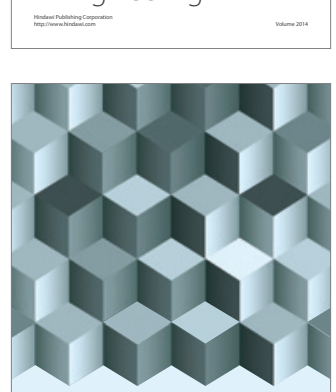

Journal of

Function Spaces
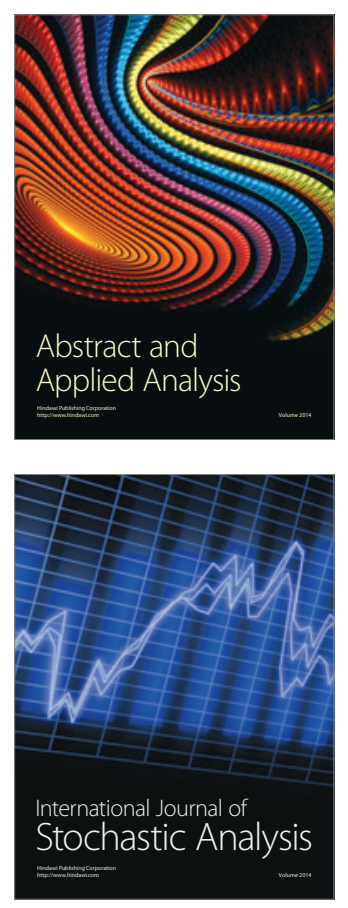

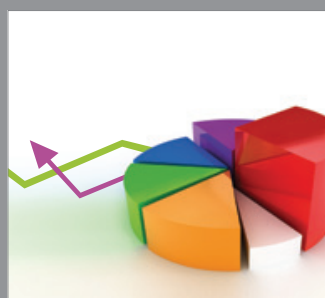

ournal of

Probability and Statistics

Promensencen
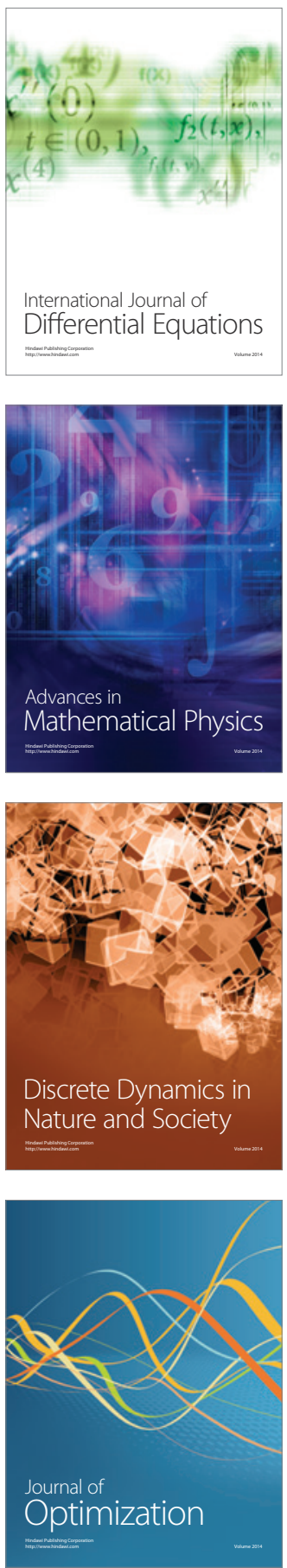